\title{
Indirect Comparison of Lanadelumab and Intravenous C1-INH Using Data from the HELP and CHANGE Studies: Bayesian and Frequentist Analyses
}

\author{
Joan Mendivil ${ }^{1}\left[\right.$ Mia Malmenäs ${ }^{2} \cdot$ Katrin Haeussler $^{3} \cdot$ Matthias Hunger $^{3} \cdot$ Gagan Jain $^{4} \cdot$ Giovanna Devercelli $^{4}$
}

Accepted: 11 January 2021 / Published online: 1 March 2021

(c) The Author(s) 2021

\begin{abstract}
Background Hereditary angioedema (HAE) with C1-esterase inhibitor (C1-INH) deficiency is a rare disease associated with painful, potentially fatal swelling episodes affecting subcutaneous or submucosal tissues. HAE attacks recur with unpredictable severity and frequency throughout patients' lives; long-term prophylaxis is essential for some patients. In the absence of head-to-head studies, indirect treatment comparison (ITC) of long-term prophylactic agents is a valid approach to evaluate comparative efficacy.

Methods We conducted an ITC using data from the placebo-controlled HELP study (assessing patients receiving lanadelumab $300 \mathrm{mg}$ every 2 or 4 weeks) and the 12-week, parallel arm, crossover CHANGE study (assessing intravenous C1-INH). Outcomes of interest were attack rate ratio (ARR) and time to attack after day 0 (TTA0) and after day 70 (TTA70). Two ITC methodologies were used: a Bayesian approach using study results to update non-informative prior distributions to posterior distributions on relative treatment effects, and a frequentist approach using patient-level data from HELP and CHANGE to generate Poisson regressions (for ARR) and Cox models (for TTA0 and TT70).

Results Both Bayesian and frequentist analyses suggested that lanadelumab reduced HAE attack rate by $46-73 \%$ versus intravenous C1-INH. Relative to intravenous C1-INH, risk of first attack after day 0 was comparable between intravenous $\mathrm{C} 1-\mathrm{INH}$ and both lanadelumab doses; risk of first attack after day 70 was reduced by $81-83 \%$ with lanadelumab $300 \mathrm{mg}$ every 2 weeks, compared with C1-INH.

Conclusions Findings from these two ITC methodologies support the favorable efficacy of lanadelumab in reducing the HAE attack rate and extending attack-free intervals in patients with HAE.
\end{abstract}

Joan Mendivil

joan.mendivil@takeda.com

1 Takeda Pharmaceuticals International AG, A Takeda Company, Zurich, Switzerland

2 ICON plc, Stockholm, Sweden

3 ICON plc, Munich, Germany

4 Takeda Pharmaceutical Company Limited, Lexington, MA, USA

\section{Key Points}

In the absence of head-to-head studies, indirect treatment comparisons provide valuable insights.

Indirect comparison of two lanadelumab dosing regimens (300 mg every 2 weeks and $300 \mathrm{mg}$ every 4 weeks) with intravenous $\mathrm{C} 1$-esterase inhibitor suggested that both lanadelumab regimens were associated with fewer hereditary angioedema attacks.

After 70 days from start of treatment (the estimated time by which steady-state lanadelumab plasma concentrations are reached), lanadelumab $300 \mathrm{mg}$ every 2 weeks was shown to extend the attack-free period. 


\section{Introduction}

Hereditary angioedema (HAE) with C1-esterase inhibitor (C1-INH) deficiency or dysfunction (HAE-1/2) is a rare genetic disease associated with recurring, unpredictable episodes of swelling affecting subcutaneous or submucosal tissues $[1,2]$. Owing to its rarity, the prevalence of HAE is unclear, but is generally regarded to be $\sim 1$ per 50,000 persons [3]. Attack severity can range from mild to moderate to severe or potentially life threatening, especially if laryngeal edema occurs $[4,5]$. Symptoms often negatively impact patients' productivity at work or school and cause emotional impairment, both during and between attacks, resulting in a substantial socioeconomic burden to patients and their families. High treatment costs, frequent use of health care resources, and absenteeism/reduced work productivity in patients with HAE are continuing challenges [5, 6]. Long-term prevention of HAE attacks may be a lifelong requirement for some patients, depending on factors such as disease activity and impact of symptoms on daily life [1].

In patients with $\mathrm{HAE}-1 / 2$, insufficient $\mathrm{C} 1$ inhibition within the kallikrein-kinin cascade leads to plasma kallikrein dysregulation, resulting in uncontrolled production of bradykinin, a potent endogenous vasodilator [7]. Owing to the impairment in C1-inhibitory mechanisms and consequent overproduction of bradykinin, replacement of C1-INH has historically been among the standard of care for long-term prophylaxis of HAE attacks.

Intravenous (IV) plasma-derived C1-INH is currently among the first-line standard-of-care options for prevention of HAE attacks $[1,8]$. However, IV administration over extended time periods may be challenging [9], and the requirement for twice-weekly dosing can be inconvenient, factors that are important to consider when individualizing therapy [1]. Of note, dosing frequency has been presumed to negatively impact patient compliance for various chronic diseases [10]. Therefore, it is a continuing goal to develop prophylactic agents that are safe, effective, and convenient to administer.

Given the critical role plasma kallikrein plays in the kallikrein-kinin cascade, this protease is an important therapeutic target in the treatment of HAE. Lanadelumab is a fully human monoclonal antibody that is a specific, potent, and long-acting inhibitor of active plasma kallikrein [11]. Efficacy and safety of subcutaneously-administered (SC) lanadelumab were demonstrated in the phase 3 , randomized, double-blind, placebo-controlled, parallel-arm HELP study (NCT02586805) [12]; recent international HAE treatment guidelines recommend this agent as a first-line treatment option for HAE attack prophylaxis [8].
Lanadelumab has been reviewed by several European Health Technology Assessment bodies (including the National Institute for Health and Care Excellence in England and Wales, the Scottish Medicine Consortium, and the Danish Medicine Council); the recommended comparator in these submissions has been IV C1-INH. To our knowledge, no head-to-head comparisons of lanadelumab with IV C1-INH have been conducted; in the absence of direct comparative trials, indirect treatment comparisons (ITCs) are a valid approach to evaluate comparative efficacy [13].

ITCs are typically developed using aggregate data from published articles or clinical trials. When individual patient data (IPD) are available, ITCs can also be conducted using patient-level data. Both Bayesian and frequentist approaches can be informed by aggregate data or IPD. IPD analyses have many advantages over aggregate analyses; they permit a greater opportunity to adjust for heterogeneity in the underlying studies in the ITC, and by doing so help to improve statistical validity $[14,15]$.

We aimed to indirectly compare lanadelumab with IV C1-INH, via both a Bayesian approach based on aggregate study results and a frequentist approach based on IPD, using data from the 26-week, placebo-controlled HELP study [12] and the 12-week, parallel-arm, crossover CHANGE study [16], to support the evidence dossier submitted to Health Technology Assessment bodies.

\section{Methods}

\subsection{ITC Overview}

The evidence network for this ITC comprises the lanadelumab $300 \mathrm{mg}$ every 2 weeks (q2w) and every 4 weeks (q4w) treatment arms from HELP [12] anchored to the IV C1-INH arm of CHANGE [16] by a common comparator (placebo) (Fig. 1). A brief overview of the design of HELP and CHANGE is presented in Electronic Supplementary Table S1 (see the electronic supplementary material), and baseline demographic and clinical characteristics for the trial arms included in this ITC are presented in Electronic Supplementary Table $\mathrm{S} 2$.

HELP was a phase 3, randomized, double-blind, placebocontrolled study using a parallel-group design and an observation time horizon (i.e., study treatment duration) of 26 weeks. Patients were randomized to receive lanadelumab $150 \mathrm{mg} \mathrm{q} 4 \mathrm{w}$, lanadelumab $300 \mathrm{mg} \mathrm{q} 4 \mathrm{w}$, lanadelumab 300 mg q $2 \mathrm{w}$, or placebo [12]. CHANGE was a phase 3, randomized, double-blind, placebo-controlled, crossover trial consisting of two 12-week periods spanning a total observation time horizon of 24 weeks. Patients were randomized to receive IV C1-INH 1000 U every 3-4 days or placebo [16]. 
Fig. 1 Evidence network for indirect treatment comparison of lanadelumab (HELP study) and IV C1-INH (CHANGE study). C1-INH C1-esterase inhibitor, $I V$ intravenous, $S C$ subcutaneous, $q 2 w$ every 2 weeks, $q 4 w$ every 4 weeks

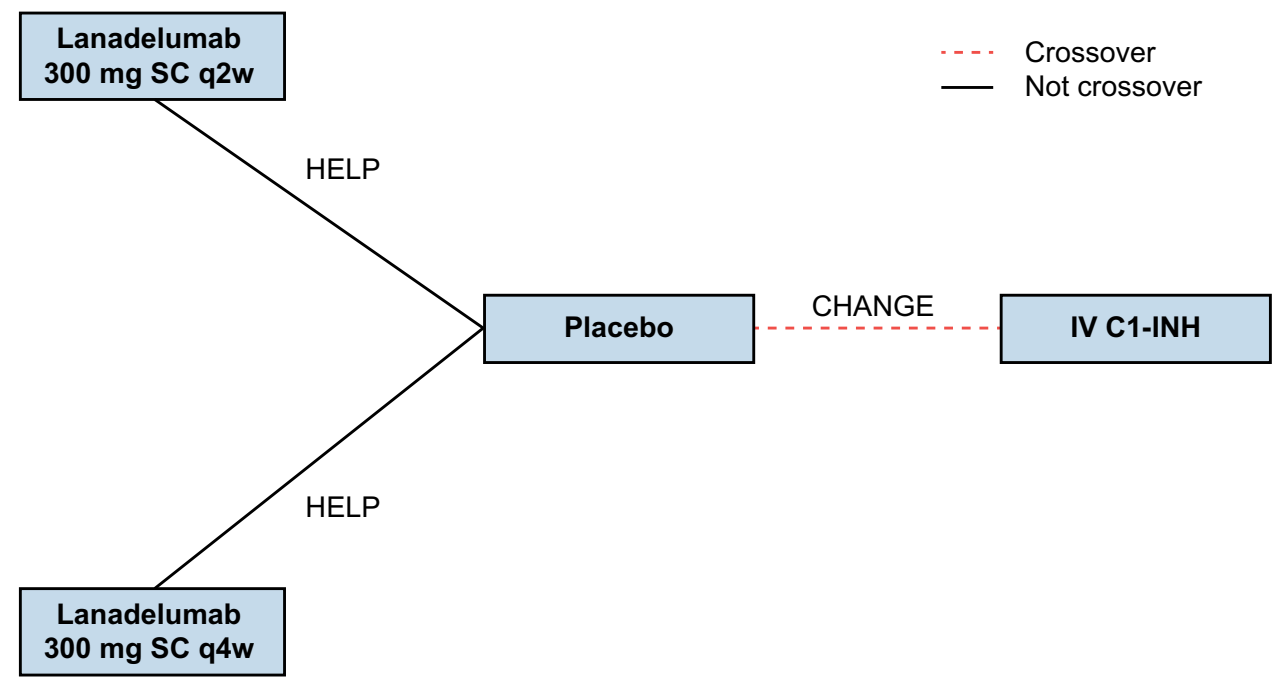

The current ITC includes only the lanadelumab $300 \mathrm{mg} \mathrm{q} 2 \mathrm{w}$ and $\mathrm{q} 4 \mathrm{w}$ arms of HELP for comparison with IV C1-INH because the recommended starting dosage of lanadelumab is $300 \mathrm{mg} \mathrm{q} 2 \mathrm{w}[17,18]$, with the option to reduce the dosage to $300 \mathrm{mg} \mathrm{q} 4 \mathrm{w}$ in patients who are stably attack free on treatment (per the lanadelumab EU Summary of Product Characteristics) or remain attack free (for at least 6 months in the Food and Drug Administration [FDA] label).

Bayesian methods are well established in ITC, in line with National Institute for Health and Care Excellence Decision Support Unit guidelines [19]. Frequentist analyses are especially suitable in cases of a sparse evidence base consisting of a limited number of trials. Both methods also assume that the included trials are fundamentally similar, preserve randomization, and can account for underlying covariates in the analysis, if the evidence base is sufficiently large. In this study, we report results from a Bayesian ITC using aggregate data from the HELP and CHANGE studies. To assess whether use of IPD yields consistent results compared with the aggregate data approach, we also conducted a frequentist analysis using IPD from both studies, following the approach taken by Bernstein et al. [20]. By using two distinct ITC methodologies, we aimed to increase certainty relating to the findings.

\subsection{Outcomes}

Outcomes of interest for this ITC included the HAE attack rate and time to first HAE attack (TTA). HAE attack rate was defined as the number of attacks experienced in a 28-day cycle, and corresponding relative treatment effects were estimated as attack rate ratios (ARRs). TTA was defined as the duration a patient with HAE remained attack free, and was measured after day 0 (first administered dose of prophylactic therapy; TTA0) and after day 70 (approximate time by which steady-state plasma lanadelumab concentration is reached
[12]; TTA70). Corresponding relative treatment effects for TTA outcomes were estimated as hazard ratios (HRs).

Notably, TTA was used as a proxy for attack-free interval, because this allowed for modeling of the results from the HELP and CHANGE studies using the same analysis. Specifically, HRs for TTA0 and TTA70 were generated using time-to-event data from HELP and binary event count data from CHANGE (i.e., the proportion of attack-free patients was used to calculate the proportion of patients experiencing at least one attack), using the method described by Woods et al. [21]. Point estimates for ARRs and HRs were developed using both ITC methodologies, and were used to derive relative treatment effects $(\%)$ between therapies.

\subsection{Bayesian ITC Methodology Based on Aggregated Data}

Bayesian Markov chain Monte Carlo methods were used to derive posterior distributions on relative treatment effects (with 95\% credible intervals [CrIs]) from non-informative prior probability distributions. In Bayesian statistics, a $\mathrm{CrI}$ is broadly equivalent to the more common confidence interval (CI) used in frequentist statistics, but is based on posterior distributions; a 95\% CrI therefore indicates that the interval has a $95 \%$ probability of containing the "true" value (in contrast, a 95\% CI suggests that the "true" value is within the interval in $95 \%$ of experimental iterations).

Fixed-effects models were chosen to perform the Bayesian ITC because no systematic differences were identified between demographic characteristics in HELP and CHANGE; it was therefore assumed that each trial was estimating the same treatment effect, and that any variation between studies was due primarily to sampling variation. Random-effects models were also tested; the corresponding deviance information criterion showed worse fit to the data than for fixed-effects models, and the corresponding $95 \%$ 
CrIs were considerably wide. This was due to the sparse evidence base and consequent large degree of uncertainty in the analysis. For lanadelumab $300 \mathrm{mg} \mathrm{q} 2 \mathrm{w}$ and $\mathrm{q} 4 \mathrm{w}$ (vs placebo), ARRs were derived from the HELP clinical study report; for IV C1-INH (vs placebo), ARRs were derived from the published data reporting mean HAE attack rate, mean difference, and CIs [16].

\subsection{Frequentist ITC Methodology Using IPD}

Poisson regression models were used to estimate HAE ARRs quantifying the relative treatment effects of lanadelumab (300 $\mathrm{mg} \mathrm{q} 2 \mathrm{w}$ and $300 \mathrm{mg} \mathrm{q} 4 \mathrm{w}$ ) and IV C1-INH relative to placebo. As observations from CHANGE were not independent, owing to the crossover design, a mixed model (including fixed factors for treatment, period, and sequence, and a random effect for study subjects) was used to account for the repeated measures. For TTA0 and TTA70, a Cox proportional hazards model was used to generate HRs for each lanadelumab dose regimen ( $300 \mathrm{mg} \mathrm{q} 2 \mathrm{w}$ and $300 \mathrm{mg} \mathrm{q} 4 \mathrm{w}$ ) relative to placebo in HELP and for IV C1-INH relative to placebo in CHANGE.

As above, mixed models (including fixed effects for the treatment, period, and sequence of crossover, and a random effect for subjects) were used to account for the crossover design of CHANGE. Kaplan-Meier curves for TTA0 and TTA70 were developed via nonparametric estimation to assess whether hazard functions remained proportional over time. Comparison between lanadelumab (300 mg q2w and $\mathrm{q} 4 \mathrm{w}$ ) and IV C1-INH was achieved using the Bucher method. The Bucher method was based on simple equations to enable an indirect comparison of active interventions via a common comparator, placebo, estimating the difference in two rate ratios with corresponding standard error on the $\log$ scale, followed by back-transformation of results. The Bucher method was informed by least-square means on treatment effects with intervention relative to placebo in each study, corresponding with the outcomes of the mixed models described above [22].

Two sensitivity analyses were conducted: sensitivity analysis 1 considered all HAE attacks reported in HELP, regardless of whether they were confirmed by an investigator; sensitivity analysis 2 included the baseline covariates of age, sex, and weight as factors in the regression model.

\section{Results}

\subsection{Descriptive Statistics from HELP and CHANGE}

Descriptive statistics for HAE attack rates, TTA0, and TTA70 derived using IPD from the HELP and CHANGE studies are presented in Table 1. It should be noted, however, that these descriptive values do not account for baseline HAE attack rate, or for the crossover effect of the CHANGE study.

\subsection{Bayesian ITC Based on Aggregated Data}

Using Bayesian methods to compare the three active interventions with placebo, ARR with lanadelumab $300 \mathrm{mg} \mathrm{q} 2 \mathrm{w}$ and $\mathrm{q} 4 \mathrm{w}$ was reduced by $87 \%$ and by $73 \%$, respectively (corresponding HRs and 95\% CrIs are presented in Fig. 2). ARR with IV C1-INH was reduced by $51 \%$, relative to placebo. Bayesian comparison of ARR with lanadelumab versus IV C1-INH demonstrated that HAE attack rates were reduced by $73 \%$ and by $46 \%$ in patients treated with lanadelumab 300 mg q2w and q4w, respectively, compared with IV C1-INH (corresponding HRs and 95\% CrIs are presented in Table 2).

Risk of first attack after day 0 was reduced by $73 \%$ and by $61 \%$ with lanadelumab $300 \mathrm{mg} \mathrm{q} 2 \mathrm{w}$ and $300 \mathrm{mg} \mathrm{q} 4 \mathrm{w}$, respectively, compared with placebo (corresponding HRs and 95\% CrIs are presented in Fig. 3a); risk of first attack after day 0 with IV C1-INH was comparable with placebo.

Table 1 Descriptive statistics for HAE attack rate and TTA outcomes in treatment arms of the HELP and CHANGE studies*

\begin{tabular}{|c|c|c|c|c|c|}
\hline & \multicolumn{3}{|l|}{ HELP } & \multicolumn{2}{|l|}{ CHANGE } \\
\hline & Placebo $(n=41)$ & $\begin{array}{l}\text { Lanadelumab q2w } \\
(n=27)\end{array}$ & $\begin{array}{l}\text { Lanadelumab q4w } \\
(n=29)\end{array}$ & Placebo $(n=22)^{\ddagger}$ & IV C1-INH $(n=22)^{*}$ \\
\hline $\begin{array}{l}\text { Mean (SD) HAE attack rate per } \\
28 \text { days during treatment }\end{array}$ & $2.45(2.08)$ & $0.31(0.50)$ & $0.60(0.80)$ & $4.24(1.55)$ & $2.09(1.85)$ \\
\hline Mean (SD) TTA0, days & $21.41(34.45)$ & $97.04(80.53)$ & $74.90(77.96)$ & $4.77(7.85)$ & $20.68(28.16)$ \\
\hline Mean (SD) TTA70, days ${ }^{\dagger}$ & $21.43(28.68)$ & $92.42(40.04)$ & $62.62(46.18)$ & $3.48(3.41)$ & $7.10(4.30)$ \\
\hline
\end{tabular}

$C 1$-INH C1-esterase inhibitor, $H A E$ hereditary angioedema, $I V$ intravenous, $q 2 w$ every 2 weeks, $q 4 w$ every 4 weeks, $S D$ standard deviation, $T T A$ time to attack, TTAO time to attack after day $0, T T A 70$ time to attack after day 70

*These descriptive values do not account for baseline HAE attack rate or for the crossover effect of the CHANGE study

Sample size for CHANGE TTA70: $n=21$

\#Due to the crossover study design, every patient is considered twice 
Fig. 2 Attack rate ratio (95\% $\mathrm{CI} / \mathrm{CrI}$ ) for all treatments vs placebo in Bayesian and frequentist analyses. Bayesian estimates are presented with CrIs; frequentist estimates are presented with CIs. C1-INH C1-esterase inhibitor, $\mathrm{CI}$ confidence interval, $\mathrm{CrI}$ credible interval, $I V$ intravenous, $q 2 w$ every 2 weeks, $q 4 w$ every 4 weeks

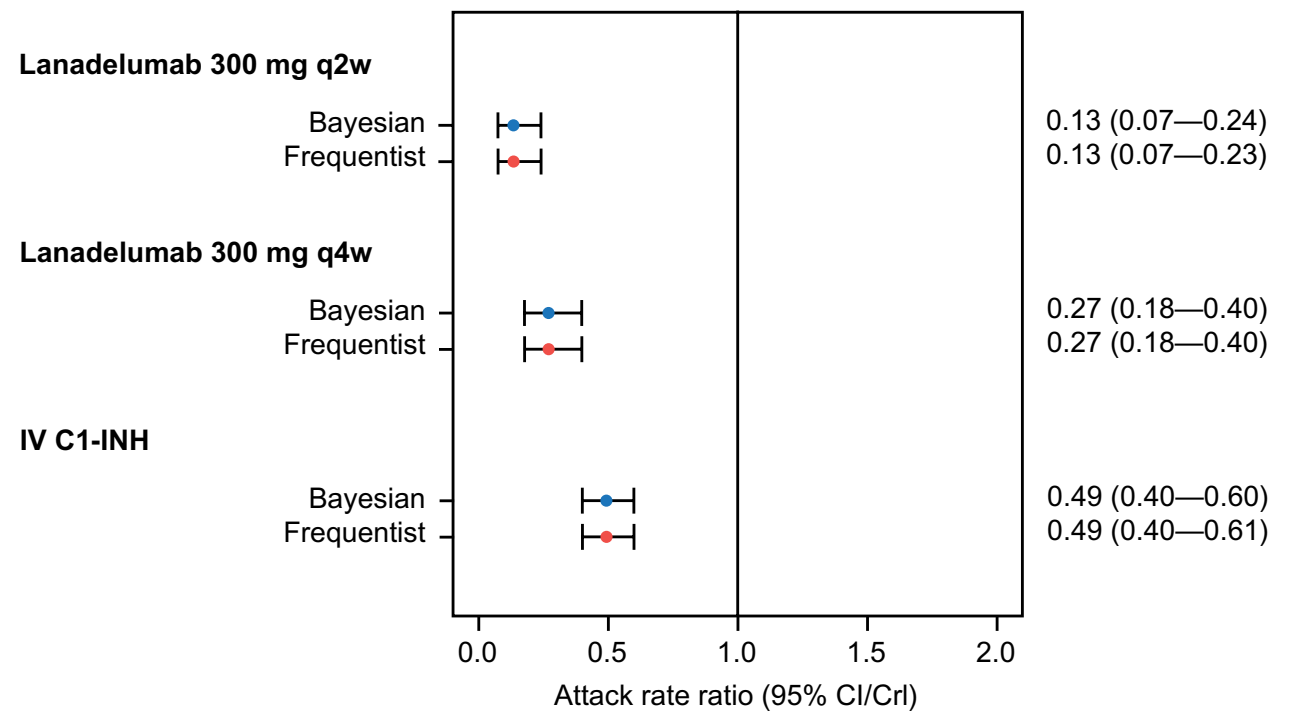

Table 2 Indirect comparison of outcomes with lanadelumab (300 $\mathrm{mg} \mathrm{q} 2 \mathrm{w}$ and $\mathrm{q} 4 \mathrm{w}$ ) vs IV C1-INH using individual patient data by the Bucher method, and by Bayesian analysis

\begin{tabular}{|c|c|c|c|c|c|c|}
\hline \multirow{2}{*}{$\begin{array}{l}\text { Comparator (vs IV } \\
\text { C1-INH) }\end{array}$} & \multicolumn{2}{|l|}{ HAE ARR } & \multicolumn{2}{|l|}{ TTA0 HR } & \multicolumn{2}{|l|}{ TTA70 HR } \\
\hline & Bucher $(95 \% \mathrm{CI})$ & Bayesian $(95 \% \mathrm{CrI})$ & Bucher $(95 \% \mathrm{CI})$ & Bayesian $(95 \% \mathrm{CrI})$ & Bucher $(95 \% \mathrm{CI})$ & Bayesian $(95 \%$ CrI $)$ \\
\hline $\begin{array}{l}\text { Lanadelumab } 300 \mathrm{mg} \\
\mathrm{q} 2 \mathrm{w}\end{array}$ & $0.27(0.15-0.49)$ & $0.27(0.14-0.51)$ & $0.73(0.29-1.84)$ & $0.51(0.22-1.32)$ & $0.19(0.06-0.62)$ & $0.17(0.05-0.57)$ \\
\hline $\begin{array}{l}\text { Lanadelumab } 300 \mathrm{mg} \\
\text { q4w }\end{array}$ & $0.54(0.35-0.85)$ & $0.54(0.34-0.86)$ & $1.05(0.43-2.52)$ & $0.73(0.26-2.09)$ & $0.62(0.23-1.64)$ & $0.51(0.18-1.49)$ \\
\hline
\end{tabular}

$A R R$ attack rate ratio, $C 1$ - $I N H$ C1-esterase inhibitor, $C I$ confidence interval, $C r I$ credible interval, $H A E$ hereditary angioedema, $H R$ hazard ratio, $I V$ intravenous, $q 2 w$ every 2 weeks, $q 4 w$ every 4 weeks, TTAO time to attack after day $0, T T A 70$ time to attack after day 70

Bayesian comparison of TTA0 HRs for lanadelumab versus IV C1-INH demonstrated that risk of first attack after day 0 with lanadelumab $300 \mathrm{mg}$ q2 $\mathrm{w}$ and $\mathrm{q} 4 \mathrm{w}$ was comparable with IV C1-INH, as indicated by the $95 \%$ CrIs (Table 2). When assessing risk of first attack after day 70 (Fig. 3b), reduction in risk of first attack with lanadelumab $300 \mathrm{mg}$ $\mathrm{q} 2 \mathrm{w}$ and $\mathrm{q} 4 \mathrm{w}$ was $91 \%$ and $73 \%$, respectively. Risk of first attack after day 70 with IV C1-INH was comparable with placebo. Per Bayesian comparison of TTA70 HRs for lanadelumab versus IV C1-INH, lanadelumab $300 \mathrm{mg}$ $\mathrm{q} 2 \mathrm{w}$ significantly reduced the risk of first attack by $83 \%$, compared with IV C1-INH (Table 2); the risk of first attack with lanadelumab $300 \mathrm{mg} \mathrm{q} 4 \mathrm{w}$ was reduced by $49 \%$ (nonsignificant, as indicated by the $95 \%$ CrIs).

\subsection{Frequentist ITC Using IPD}

Review of the Kaplan-Meier model for TTA data in HELP did not suggest that the assumption of proportional hazards should be rejected (i.e., the curves for lanadelumab remained broadly parallel to, and did not cross, the curve for placebo) (Electronic Supplementary Fig. S1). Compared with placebo, ARR was significantly reduced by $87 \%$ and by $73 \%$ with lanadelumab $300 \mathrm{mg} \mathrm{q} 2 \mathrm{w}$ and q4w, respectively (corresponding HRs and 95\% CIs are presented in Fig. 2). ARR relative to placebo was also statistically significantly reduced by $51 \%$ with IV C1-INH. Bucher comparison of HAE ARRs demonstrated that HAE attack rates were statistically significantly reduced by $73 \%$ and by $46 \%$ in patients treated with lanadelumab $300 \mathrm{mg} \mathrm{q} 2 \mathrm{w}$ and $\mathrm{q} 4 \mathrm{w}$, respectively, compared with IV C1-INH (corresponding HRs and 95\% CIs are presented in Table 2).

Compared with placebo, risk of first attack after day 0 was reduced by $73 \%$ and $62 \%$ with lanadelumab $300 \mathrm{mg} \mathrm{q} 2 \mathrm{w}$ and $\mathrm{q} 4 \mathrm{w}$, respectively, and by $63 \%$ with IV C1-INH (Fig. 3a). When evaluating TTA0 HRs by the Bucher method, lanadelumab $300 \mathrm{mg}$ q2 $\mathrm{w}$ and $\mathrm{q} 4 \mathrm{w}$ did not appear to significantly reduce the risk of first attack compared with IV C1-INH, as indicated by the $95 \%$ CIs (Table 2). Relative to placebo, risk of first attack after day 70 was reduced by $93 \%$ and $79 \%$ with lanadelumab $300 \mathrm{mg} \mathrm{q} 2 \mathrm{w}$ and $\mathrm{q} 4 \mathrm{w}$, respectively, and by $66 \%$ with IV C1-INH (Fig. 3b). When comparing TTA70 HRs by the Bucher method, $300 \mathrm{mg} \mathrm{q} 2 \mathrm{w}$ significantly reduced the risk of first attack after day 70 by $81 \%$ (Table 2); the risk of 
Fig. 3 Time to first attack HR (95\% CI/CrI) for all treatments vs placebo after day 0 (a) and day 70 (b). Bayesian estimates are presented with CrIs; frequentist estimates are presented with CIs. C1-INH C1-esterase inhibitor, $C I$ confidence interval, $C r I$ credible interval, $H R$ hazard ratio, $I V$ intravenous, $q 2 w$ every 2 weeks, $q 4 w$ every 4 weeks, TTAO time to attack after day $0, T T A 70$ time to attack after day 70

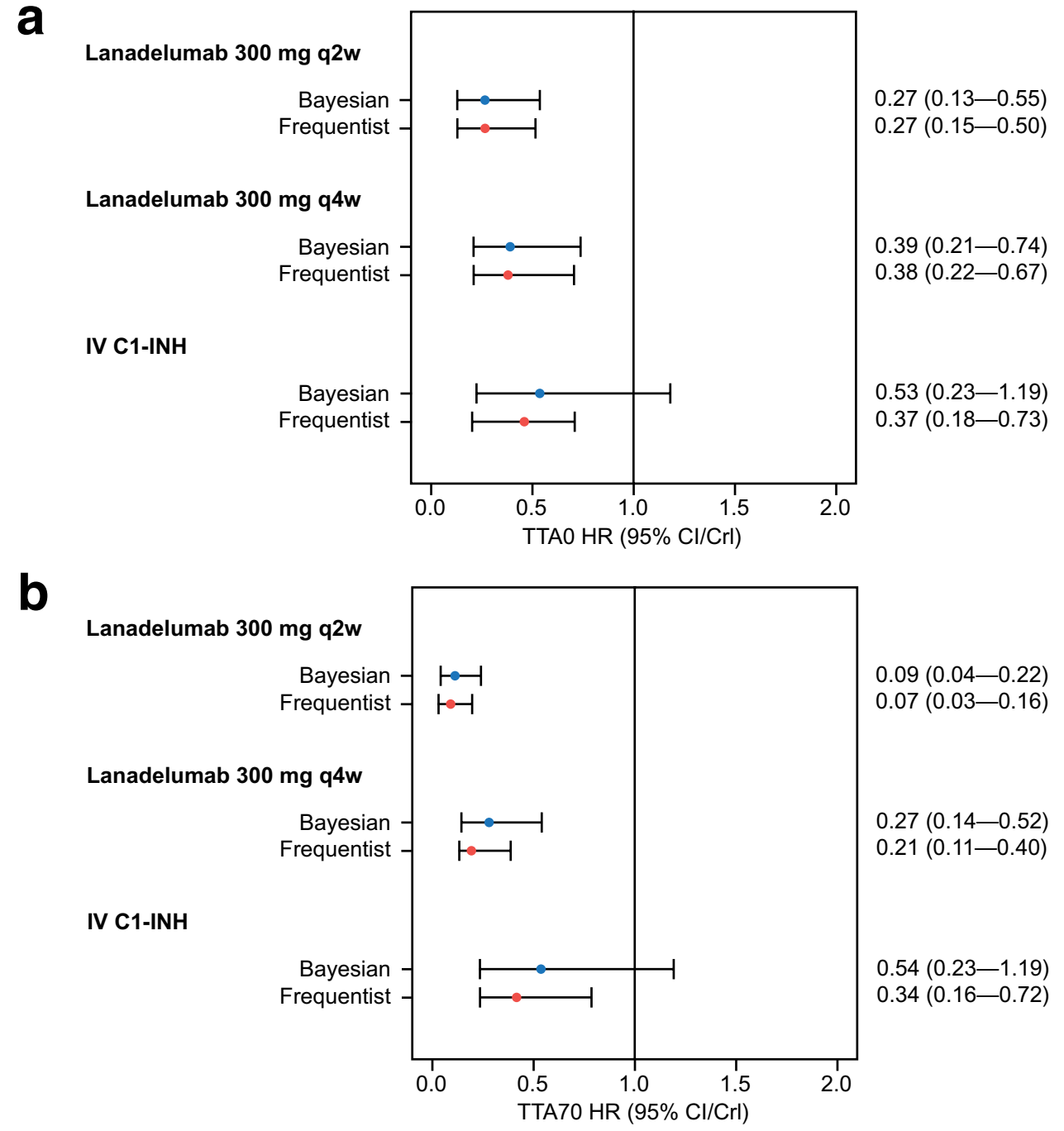

first attack with lanadelumab $300 \mathrm{mg} \mathrm{q} 4 \mathrm{w}$ was reduced by $38 \%$ (non-significant, as indicated by the $95 \%$ CIs).

In sensitivity analyses considering all reported HAE attacks in the HAE ARR analysis-and also considering the baseline covariates of age, gender, and weight as factors in the regression models-Bucher comparisons of lanadelumab (both dose regimens) and IV C1-INH were nearly identical to the primary analysis (Electronic Supplementary Table S3).

\section{Discussion}

The results of this ITC, using both Bayesian and frequentist approaches, were consistent: compared with placebo, lanadelumab $300 \mathrm{mg} \mathrm{q} 2 \mathrm{w}$, lanadelumab $300 \mathrm{mg} \mathrm{q} 4 \mathrm{w}$, and IV C1-INH were associated with reduced HAE attack rate and reduced risk of first HAE attack in patients with HAE. Bayesian and frequentist approaches both indicated that lanadelumab dosages were associated with a favorable and statistically significant reduction in HAE attack rate compared with IV C1-INH, and lanadelumab $300 \mathrm{mg} \mathrm{q} 2 \mathrm{w}$ was also associated with statistically significant reductions in the risk of first attack after day 70 compared with IV C1-INH.

By using TTA as a proxy for attack-free interval, this analysis permitted comparison of time-to-event data from HELP with event count data from CHANGE. Although most HAE point estimates were nearly identical for Bayesian and frequentist analyses, most of the frequentist estimates for TTA0 and TTA70 were slightly lower than for Bayesian methods. This may reflect the use of a mixed model to account for the repeated measures seen within patients in the crossover CHANGE study.

Risk of first attack with lanadelumab in the TTA70 analysis was substantially lower than in the TTA0 analysis; this aligns with the known time to steady-state concentration of lanadelumab [12]. Accordingly, post hoc analyses from HELP have demonstrated that HAE attack rate and 
proportion of patients who remain attack-free improve with lanadelumab within 0-69 days (vs placebo), but further improvement in these outcomes was seen from 70 days onward [23]. More recently, efficacy of long-term prophylactic therapy with lanadelumab $300 \mathrm{mg}$ q2w was demonstrated in the HELP open-label extension study [24]. Although assessment of the cost implications of this reduction in resource use with long-term lanadelumab is interesting to consider, such comparison is beyond the scope of the present study.

The route of administration is an important practical consideration for both physicians and patients [9]. Self-administration of medication has been reported to be associated with patient satisfaction and medication compliance [25]; and according to a survey of US patients with HAE, the most common barrier to self-administration is a fear of injections [26]. In addition to the efficacy advantage seen with SC administration in this ITC, lanadelumab has the advantage of being easier to self-administer than C1-INH, which may help improve patient compliance. Accordingly, an interim analysis from the HELP open-label extension suggested that the majority of patients who self-administered SC lanadelumab and were experienced with prior use of IV C1-INH for longterm prophylaxis preferred SC administration (70.9\% of 55 patients), with $59.3 \%$ and $52.5 \%$ of 59 patients reporting that $\mathrm{SC}$ injections were easier to administer and convenient, respectively [27]. Of note, an SC C1-INH formulation was recently approved by the FDA and European Medicines Agency for prophylaxis of HAE attacks. This formulation requires dosing every 3-4 days [28].

The current ITC analysis was conducted using two rigorous ITC methodologies: a more frequently used Bayesian method, generally recognized by Health Technology Assessment authorities; and a patient-level frequentist approach, which facilitates estimation of subgroup effects, aids convergence, and yields more precise estimates overall [14, 15]. Point estimates obtained via the frequentist method are generally very similar to those obtained through Bayesian approaches; however, CIs are usually narrower than the CrIs of a Bayesian analysis when based on sparse evidence, with non-informative priors assigned to treatment effects [29]. Additionally, IPD allow within-study associations to be distinguished from across-study associations [13, 15] and can reduce ecological bias [13]. In this analysis, results from sensitivity analyses accounting for age, gender, and weight were numerically and qualitatively consistent with findings from the primary analysis.

Of note, this ITC excluded the lanadelumab $150 \mathrm{mg}$ $\mathrm{q} 4 \mathrm{w}$ arm of HELP. This is in line with current market authorizations for lanadelumab, including the approved labeling in the United States [18] and the EU [17], which recommend a starting dose of $300 \mathrm{mg} \mathrm{q} 2 \mathrm{w}$ with a potential dose reduction to $300 \mathrm{mg} \mathrm{q} 4 \mathrm{w}$ in patients with well-controlled disease. Omitting the lanadelumab $150 \mathrm{mg}$ $\mathrm{q} 4 \mathrm{w}$ dosing group also mitigated the multiplicity errors that can occur in frequentist analyses when multiple statistical comparisons are made.

Limitations of our methodology include those inherent to any ITC. Although HELP and CHANGE were broadly similar in terms of age, sex, and number of enrolled patients with HAE, residual heterogeneity in the included trials may have influenced the findings. Such limitations should be considered when interpreting findings from ITCs, which are not a substitute for head-to-head comparisons [13]. As our ITC is based on data obtained from double-blind, placebo-controlled, randomized clinical studies, the potential impact of off-label uptitrated doses of IV C1-INH on effectiveness was not considered. Additionally, the HELP and CHANGE studies contained small samples sizes (owing to the rarity of the disease), which impeded the statistical power of the frequentist analysis. In Bayesian ITC, a sparse evidence base comprising few studies with few interventions introduces a large degree of uncertainty that can lead to wide CrIs, especially if non-informative priors are assigned to treatment effects. Lastly, CHANGE did not provide patient-level data for TTA0 and TTA70; HRs for TTA analyses were generated using binary data (the proportion of attack-free patients was used to calculate the proportion of patients experiencing at least one attack).

In conclusion, this analysis used two established, complementary ITC methodologies to compare the clinical outcomes of two lanadelumab dosing regimens with those of IV C1-INH. The findings from these respective methodologies are consistent, and support the efficacy of lanadelumab in reducing HAE attack rate and extending attack-free periods in patients with HAE.

Supplementary Information The online version contains supplementary material available at https://doi.org/10.1007/s40268-021-00337-4.

Acknowledgements Under direction of the authors, Martin Bell, Ph.D., and Sophia Shumyatsky, PharmD, CMPP, employees of Excel Medical Affairs, provided writing assistance for this article. Shire Human Genetic Therapies, Inc., a Takeda company, provided funding to Excel Medical Affairs for support in writing and editing this article. The interpretation of the data was made by the authors independently. Richard Birnie contributed to the design and data analysis of an early version of the Bayesian ITC.

\section{Declarations}

Funding This ITC was sponsored by Shire Human Genetic Therapies, Inc., a Takeda company, Lexington, MA, USA.

Conflict of interest Joan Mendivil, Gagan Jain, and Giovanna Devercelli are full-time employees of Takeda. Mia Malmenäs, Katrin Haeussler, and Matthias Hunger are full-time employees of ICON plc. 
Ethics approval Not applicable.

Consent to participate Not applicable.

Consent for publication Not applicable.

Data Availability The datasets supporting the results reported in this article will be available 3 months after the submission of a request to researchers who provide a methodologically sound proposal. The data will be provided after its de-identification, in compliance with applicable privacy laws, data protection, and requirements for consent and anonymization.

\section{Code availability Not applicable.}

Author contributions JM and $\mathrm{KH}$ contributed to data acquisition. All authors contributed to study design, data analysis and/or interpretation, drafting of the manuscript or revising for important intellectual content, and approval of the final draft.

Open Access This article is licensed under a Creative Commons Attribution-NonCommercial 4.0 International License, which permits any non-commercial use, sharing, adaptation, distribution and reproduction in any medium or format, as long as you give appropriate credit to the original author(s) and the source, provide a link to the Creative Commons licence, and indicate if changes were made. The images or other third party material in this article are included in the article's Creative Commons licence, unless indicated otherwise in a credit line to the material. If material is not included in the article's Creative Commons licence and your intended use is not permitted by statutory regulation or exceeds the permitted use, you will need to obtain permission directly from the copyright holder. To view a copy of this licence, visit http://creativecommons.org/licenses/by-nc/4.0/.

\section{References}

1. Maurer M, Magerl M, Ansotegui I, Aygören-Pürsün E, Betschel $\mathrm{S}$, Bork $\mathrm{K}$, et al. The international WAO/EAACI guideline for the management of hereditary angioedema-the 2017 revision and update. Allergy. 2018;73(8):1575-96. https://doi.org/10.1111/ all.13384.

2. Lang DM, Aberer W, Bernstein JA, Chng HH, Grumach AS, Hide $\mathrm{M}$, et al. International consensus on hereditary and acquired angioedema. Ann Allergy Asthma Immunol. 2012;109(6):395-402. https://doi.org/10.1016/j.anai.2012.10.008.

3. Zuraw BL, Banerji A, Bernstein JA, Busse PJ, Christiansen SC, Davis-Lorton M, et al. US Hereditary Angioedema Association Medical Advisory Board 2013 recommendations for the management of hereditary angioedema due to $\mathrm{C} 1$ inhibitor deficiency. J Allergy Clin Immunol Pract. 2013;1(5):458-67. https://doi. org/10.1016/j.jaip.2013.07.002.

4. Bork K, Meng G, Staubach P, Hardt J. Hereditary angioedema: new findings concerning symptoms, affected organs, and course. Am J Med. 2006;119(3):267-74. https://doi.org/10.1016/j.amjme d.2005.09.064.

5. Banerji A, Davis KH, Brown TM, Hollis K, Hunter SM, Long $\mathrm{J}$, et al. Patient-reported burden of hereditary angioedema: findings from a patient survey in the United States. Ann Allergy Asthma Immunol. 2020;124(6):600-7. https://doi.org/10.1016/j. anai.2020.02.018 ((Epub ahead of print)).

6. Aygören-Pürsün E, Bygum A, Beusterien K, Hautamaki E, Sisic Z, Wait S, et al. Socioeconomic burden of hereditary angioedema: results from the hereditary angioedema burden of illness study in Europe. Orphanet J Rare Dis. 2014;9:99. https:// doi.org/10.1186/1750-1172-9-99.

7. Kaplan AP, Joseph K. Complement, kinins, and hereditary angioedema: mechanisms of plasma instability when $\mathrm{C} 1$ inhibitor is absent. Clin Rev Allergy Immunol. 2016;51(2):207-15. https:// doi.org/10.1007/s12016-016-8555-6.

8. Betschel S, Badiou J, Binkley K, Borici-Mazi R, Hébert J, Kanani A, et al. The International/Canadian Hereditary Angioedema Guideline. Allergy Asthma Clin Immunol. 2019;15:72. https:// doi.org/10.1186/s13223-019-0376-8.

9. Riedl MA, Banerji A, Busse PJ, Johnston DT, Davis-Lorton MA, Patel S, et al. Patient satisfaction and experience with intravenously administered $\mathrm{C} 1$-inhibitor concentrates in the United States. Ann Allergy Asthma Immunol. 2017;119(1):59-64. https ://doi.org/10.1016/j.anai.2017.05.017.

10. Ingersoll KS, Cohen J. The impact of medication regimen factors on adherence to chronic treatment: a review of literature. J Behav Med. 2008;31(3):213-24. https://doi.org/10.1007/s1086 5-007-9147-y.

11. Kenniston JA, Faucette RR, Martik D, Comeau SR, Lindberg AP, Kopacz KJ, et al. Inhibition of plasma kallikrein by a highly specific active site blocking antibody. J Biol Chem. 2014;289(34):23596-608. https://doi.org/10.1074/jbc. M114.569061.

12. Banerji A, Riedl MA, Bernstein JA, Cicardi M, Longhurst HJ, Zuraw BL, et al. Effect of lanadelumab compared with placebo on prevention of hereditary angioedema attacks: a randomized clinical trial. JAMA. 2018;320(20):2108-21. https://doi.org/10.1001/ jama.2018.16773.

13. Jansen JP, Fleurence R, Devine B, Itzler R, Barrett A, Hawkins $\mathrm{N}$, et al. Interpreting indirect treatment comparisons and network meta-analysis for health-care decision making: report of the ISPOR Task Force on Indirect Treatment Comparisons Good Research Practices: part 1. Value Health. 2011;14(4):417-28. https://doi.org/10.1016/j.jval.2011.04.002.

14. Saramago P, Sutton AJ, Cooper NJ, Manca A. Mixed treatment comparisons using aggregate and individual participant level data. Stat Med. 2012;31(28):3516-36. https://doi.org/10.1002/ sim.5442.

15. Riley RD, Lambert PC, Staessen JA, Wang J, Gueyffier F, Thijs L, et al. Meta-analysis of continuous outcomes combining individual patient data and aggregate data. Stat Med. 2008;27(11):1870-93. https://doi.org/10.1002/sim.3165.

16. Zuraw BL, Busse PJ, White M, Jacobs J, Lumry W, Baker J, et al. Nanofiltered C1 inhibitor concentrate for treatment of hereditary angioedema. N Engl J Med. 2010;363(6):513-22. https://doi. org/10.1056/NEJMoa0805538.

17. European Medicines Agency. TAKHZYRO $300 \mathrm{mg}$ solution for injection. 2020. https://www.ema.europa.eu/en/documents/ product-information/takhzyro-epar-product-information_en.pdf. Accessed 1 May 2020.

18. US Food and Drug Administration. TAKHZYRO ${ }^{\mathrm{TM}}$ (lanadelumab-flyo) injection, for subcutaneous use. 2018. https://www. accessdata.fda.gov/drugsatfda_docs/label/2018/761090s000lbl. pdf. Accessed 1 May 2020.

19. Dias S, Welton NJ, Sutton AJ, Ades AE. NICE DSU Technical Support Document 2: a generalised linear modelling framework for pairwise and network meta-analysis of randomised controlled trials. London: National Institute for Health and Care Excellence (NICE); 2014.

20. Bernstein JA, Li HH, Craig TJ, Manning ME, Lawo JP, Machnig $\mathrm{T}$, et al. Indirect comparison of intravenous vs. subcutaneous C1-inhibitor placebo-controlled trials for routine prevention of hereditary angioedema attacks. Allergy Asthma Clin Immunol. 2019;15:13. https://doi.org/10.1186/s13223-019-0328-3. 
21. Woods BS, Hawkins N, Scott DA. Network meta-analysis on the log-hazard scale, combining count and hazard ratio statistics accounting for multi-arm trials: a tutorial. BMC Med Res Methodol. 2010;10:54. https://doi.org/10.1186/1471-2288-10-54.

22. Bucher HC, Guyatt GH, Griffith LE, Walter SD. The results of direct and indirect treatment comparisons in meta-analysis of randomized controlled trials. J Clin Epidemiol. 1997;50:683-91.

23. Riedl MA, Maurer M, Bernstein JA, Banerji A, Longhurst $\mathrm{HJ}, \mathrm{Li} \mathrm{HH}$, et al. Lanadelumab demonstrates rapid and sustained prevention of hereditary angioedema attacks. Allergy. 2020;75(11):2879-87. https://doi.org/10.1111/all.14416.

24. Riedl M, Johnston D, Lumry W, Bernstein J, Nurse C, Lu P, et al. Attack-free status of patients with hereditary angioedema (HAE) during extended treatment with lanadelumab in the HELP OLEstudy. J Allergy Clin Immunol. 2020;145(2, Supplement):AB104. https://doi.org/10.1016/j.jaci.2019.12.626.

25. Wang A, Fouche A, Craig TJ. Patients perception of self-administrated medication in the treatment of hereditary angioedema.
Ann Allergy Asthma Immunol. 2015;115(2):120-5. https://doi. org/10.1016/j.anai.2015.06.006.

26. Tuong LA, Olivieri K, Craig TJ. Barriers to self-administered therapy for hereditary angioedema. Allergy Asthma Proc. 2014;35(3):250-4. https://doi.org/10.2500/aap.2014.35.3753.

27. Lumry W, Busse P, Lu P, Jain G, Nurse C, Maurer M. Subcutaneous self-administration of lanadelumab for prophylactic treatment in patients with hereditary angioedema (HAE). Ann Allergy Asthma Immunol. 2018;121(5, Supplement):S57. https ://doi.org/10.1016/j.anai.2018.09.186.

28. US Food and Drug Administration. HAEGARDA full prescribing information. 2020. https://www.fda.gov/media/105611/download. Accessed 27 Nov 2020.

29. Biondi-Zoccai G. Network meta-analysis: evidence synthesis with mixed treatment comparison. New York: Nova Science Publishers; 2014. 